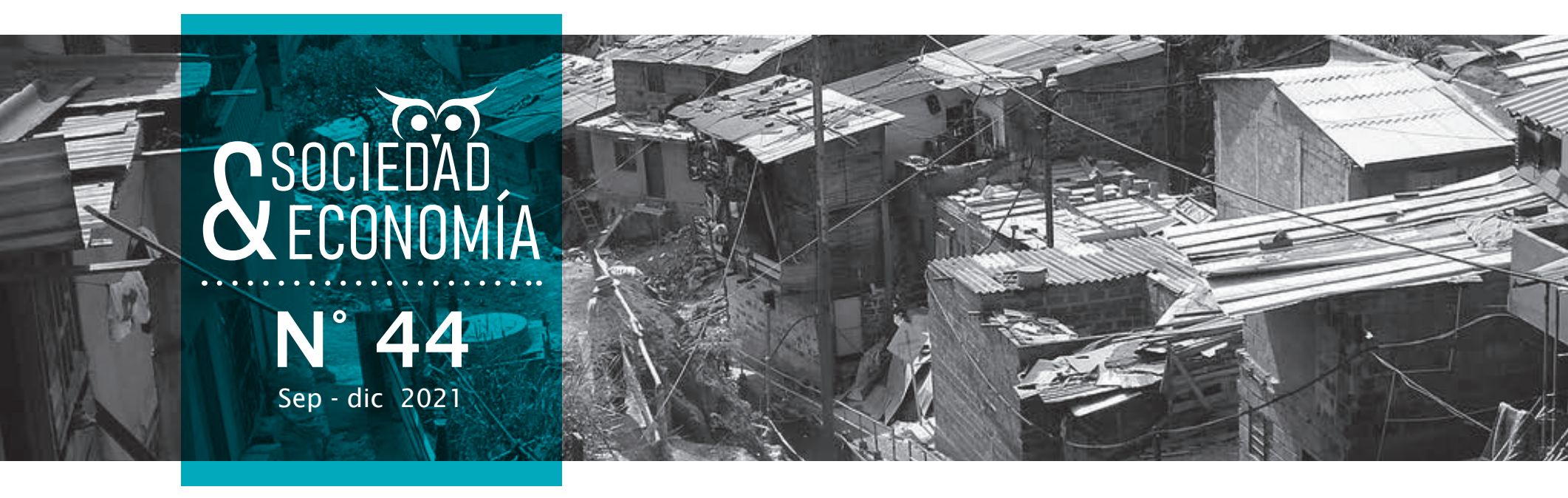

\title{
Perspectiva multidimensional de la pobreza en los hogares colombianos
}

\author{
Multidimensional Perspective of Poverty in Colombian Households
}

\author{
Edith Johana Medina-Hernández'1 \\ Universidad de Salamanca, Salamanca, España \\ 실.medina@usal.es \\ (1) https://orcid.org/0000-0003-4207-0333 \\ Luis Francisco Sierra-Ibáñez ${ }^{2}$ \\ Colegio Salesiano San Pedro Claver de Cartagena, Cartagena, Colombia \\ Q1uis.sierra@salesianoscartagena.edu.co \\ (1) https://orcid.org/0000-0003-4745-8978
}

\section{Andy Rafael Domínguez-Monterrosa ${ }^{3}$}

Universidad Tecnológica de Bolívar, Cartagena, Colombia

@adominguez@utb.edu.co

(D) https://orcid.org/0000-0002-5274-7443
Recibido: 07-1 1-2020 Aceptado: 26-02-2021 Publicado: 01-09-2021

1 Magíster en Análisis Avanzado de Datos Multivariantes.

2 Magíster en Estadística Aplicada.

3 Magíster en Matemáticas Aplicadas. 


\title{
Resumen
}

En este estudio, se analizan los últimos datos del Índice de Pobreza Multidimensional (IPM) en Colombia, publicados por el Departamento Administrativo Nacional de Estadística (DANE); examinando privaciones en los hogares del país y variables sociodemográficas de las personas que los integran. Se busca caracterizar los factores condicionantes de la pobreza según regiones, a través de un análisis cuantitativo de carácter descriptivo y mediante la aplicación de la técnica multivariante: Análisis de Componentes Principales (PCA). Los resultados obtenidos muestran que variables como el número de integrantes del hogar, su logro educativo y el acceso al empleo formal, determinan diferencias considerables entre los hogares considerados como pobres y no pobres. Adicionalmente, se observa preponderancia de circunstancias de pobreza en las regiones costeras del país y la región amazónica.

Palabras clave: pobreza; análisis multivariado; investigación demográfica; política pública.

Clasificación JEL: I32, C30, C65, J11, I38.

\begin{abstract}
This study analyzes the latest data of the Multidimensional Poverty Index (IPM) in Colombia, published by the National Administrative Department of Statistics (DANE); examining deprivations in the country's households and sociodemographic variables of the people who are part of them. The aim is to characterize the conditioning factors of poverty according to regions, through a descriptive quantitative analysis and by applying the multivariate technique: Principal Component Analysis (PCA). The results obtained show that variables such as the number of household members, their educational attainment and access to formal employment determine considerable differences between households considered poor and non-poor. In addition, a preponderance of poverty circumstances is observed in the coastal regions of the country and the Amazon region.
\end{abstract}

Keywords: poverty; multivariate analysis; demographic research; public policy.

JEL Classification: 132, C30, C65, J11, I38.

\section{Financiación}

Este trabajo no tuvo ningún tipo de financiamiento institucional.

\section{Conflicto de interés}

Los autores declaran no tener ningún conflicto de interés en la publicación de este artículo. 


\section{Introducción}

¿Por qué estudiar los factores o condiciones que determinan la pobreza asumiendo que es un fenómeno multidimensional?, ¿por qué hacerlo analizando los hogares colombianos?, y ¿por qué buscar si existen diferencias estadísticamente significativas por regiones? Estas son las preguntas articuladoras por responder para contextualizar esta investigación.

La pobreza es un fenómeno social, y específicamente en el contexto de los países latinoamericanos, o de todos los países en vía de desarrollo, afecta la economía de las naciones. Munévar (2019) afirma al respecto: "la pobreza y exclusión social de los países latinoamericanos es un problema persistente en el contexto de la economía mundial" (p. 1). Por ello, es importante entender las posibles causas y consecuencias de esta problemática que afecta a la economía doméstica.

En la mayoría de los países latinoamericanos, la población tiene elevados índices de privación en el acceso a condiciones adecuadas de empleabilidad formal, alimentación, servicios de salud y educación, que llevan a las personas a ser consideradas pobres. Esta condición no significa únicamente tener limitaciones económicas o ingresos básicos de subsistencia por encima de cierto umbral, porque, como especifican Gamboa et al. (2020), "la pobreza se conceptualiza cada vez más como un fenómeno que implica la privación en diferentes dimensiones" (p. 1).

Tejero-Pérez (2018), analizando la relación existente entre la pobreza y el acceso al empleo formal, sostiene que "los estudios sobre pobreza han mostrado la relevancia que tiene la participación laboral de los miembros adultos del hogar en la probabilidad de caer o de evitar dicha situación" (p. 2). No obstante, acceder al mercado del trabajo no debe considerarse como una condición suficiente para evitar la pobreza.

Sacchidanand y Biswas (2017) argumentan frente a la relación entre la pobreza y la educación formal, que "el número de miembros alfabetizados o la presencia de un miembro gra- duado o con educación superior en el hogar, disminuye la probabilidad de que sea pobre. El aumento de la inversión en educación puede llevar a un hogar de ser pobre a no pobre" ( $p$. 151). Por lo cual, la inversión en educación de calidad contribuye a disminuir las condiciones de pobreza en los hogares, porque con ella se mejoran los medios para satisfacer las necesidades básicas, disminuyendo la carencia de recursos.

Rajeev (2012) relaciona la pobreza con las posibilidades de adquisición de los alimentos en el hogar, cuando argumenta: "fundamentalmente, para grandes segmentos de la población mundial, las raíces del problema del hambre y la desnutrición no es la falta de alimentos, sino la falta de acceso a alimentos disponibles y adecuados, entre otros, debido a la pobreza" (p. 155). Así pues, la falta de alimentación apropiada es otro de los factores condicionantes de la pobreza, porque, al menos en el caso colombiano, es común entre los hogares cuyos integrantes no cuentan con un empleo formal o no tienen mayores logros educativos; que no puedan tener una buena alimentación y, como consecuencia, en tales hogares prevalecen situaciones de pobreza.

En razón a lo expuesto, diversos factores condicionan la pobreza de las personas y diversas metodologías se han implementado para buscar cuantificarla. Una de las más utilizadas en el contexto internacional es la de Alkire y Foster (2007), que considera el cálculo de un indicador multidimensional compuesto por distintos factores de privación de los hogares. Según como especifica Corbelle-Cacabelos y Troitiño-Cobas (2021), "esta metodología es referente para el análisis de la pobreza, especialmente por su capacidad para desagregar la pobreza por dimensiones, por características personales, del hogar o geográficas" (p. 57).

También el Índice de Desarrollo Humano (IDH) y el Índice de Necesidades Básicas Insatisfechas (NBI), se consideran como referentes para la medición y comparación de la pobreza entre países del mundo, pero desde un enfoque de capacidades y bienestar. Tanto el IPM, como el IDH y el NBI, no se limitan a considerar la 
condición de ser pobre o no, según valores económicos, porque precisamente otro enfoque para la cuantificación de la pobreza es justamente el monetario. Según como especifica Fagnola y Moneta-Pizarro (2021), "no se puede negar la ventaja de la simplicidad del enfoque unidimensional de la pobreza monetaria, particularmente cuando los hacedores de políticas públicas requieren información periódica para poder proveer de soluciones rápidas a la población" (p. 5). Sin embargo, la pobreza es un fenómeno estructural e involucra múltiples dimensiones.

Específicamente en Colombia, la fuente oficial de datos sobre la pobreza multidimensional y monetaria es el Departamento Administrativo Nacional de Estadística (DANE) y, en consecuencia, para el desarrollo de este análisis, se utiliza la última información oficial publicada en su web de microdatos. Específicamente se analiza la información de hogares y personas, registrados en la base de datos del Índice de Pobreza Multidimensional (DANE, 2019), con el fin de llegar a una conclusión respecto a cuáles son las variables que determinan la pobreza en el país, cómo estas se asocian entre sí y cuáles generan diferencias por regiones.

El interés por analizar diferencias entre territorios radica en estudios y noticias que exponen disparidades por zonas geográficas. Por ejemplo, González (2019), analizando la tendencia del IPM en Colombia del 2010 al 2018, encuentra que mientras en el Caribe y el Pacífico la incidencia de la pobreza multidimensional supera el $33 \%$, en Bogotá es del 4,3\%, afirmando que estas desigualdades no disminuyeron en el periodo analizado, porque tanto en el 2010 como en el 2018 , las dos regiones con mayor prevalencia de población pobre son las costeras.

En consecuencia de lo anterior, el principal objetivo de esta investigación es analizar los factores determinantes de la pobreza en los hogares colombianos, a partir de datos de la encuesta IPM (DANE, 2019), identificando diferencias y similitudes por regiones. Como objetivos específicos, se busca caracterizar los hogares según si son considerados pobres o no, determinando los principales factores que condicio- nan esta clasificación. Adicionalmente, se busca concluir sobre aspectos útiles para el diseño de estrategias y acciones de políticas públicas que busquen disminuir la pobreza a nivel nacional y/o regional.

A diferencia de las propuestas que internacionalmente se utilizan para medir y describir la pobreza de forma multidimensional en los hogares, como la de Alkire y Foster (2007), el análisis que se propone en esta investigación combina información de privaciones en los hogares y características de las personas que los integran, mientras que el IPM solo considera características de los hogares. Esto constituye una de las ventajas o diferenciales de este estudio frente a otras investigaciones de la pobreza en el país. Puntualmente se estudian géneros, edades y niveles educativos de las personas. En contraste, la metodología propuesta no necesariamente podría aplicarse en otros países, por ejemplo latinoamericanos, puesto que en sus mediciones del IPM, podrían no incluirse las variables de personas que aquí resultan de interés.

Para terminar estas notas introductorias, es menester indicar la estructura organizativa de este documento. Inicialmente, se presentan aspectos metodológicos de la investigación definiendo las variables y muestra en estudio, para posteriormente señalar aspectos técnicos de la técnica estadística usada para obtener los resultados. Estos últimos, se estructuran desde una exploración descriptiva de variables individuales que caracterizan la pobreza en los hogares colombianos, junto a resultados de correlaciones bivariadas y asociaciones multivariantes resultantes de un análisis de componentes principales. Se finaliza discutiendo los principales hallazgos de la investigación.

\section{Antecedentes}

Antes de presentar los aspectos metodológicos del estudio, es conveniente delimitar a modo de antecedentes, el ámbito legal histórico en el que se enmarca esta investigación, y referenciar investigaciones que fundamentan el análisis de las condiciones de pobreza desde técnicas cuantitativas de análisis de datos, específicamente en el contexto latinoamericano y de Colombia. 
En cuanto al primer aspecto, dos de las leyes que recientemente ha implementado el gobierno nacional para hacer frente a la superación de la pobreza en el país son la ley 1785 del 21 de junio 2016 (Congreso de la República de Colombia, 2016) y la ley 1948 del 08 de enero 2019 (Congreso de la República de Colombia, 2019). La primera tiene como objeto establecer una red de instituciones para la superación de la pobreza extrema, facultando el funcionamiento de la denominada Red Unidos. Esta red está conformada por las entidades del Estado que prestan servicio social a las personas en extrema pobreza, como lo son las alcaldías y gobernaciones, el sector privado y las organizaciones de la sociedad civil.

La segunda ley, por su parte, se asocia a la creación del programa Familias en Acción, el cual consiste en la entrega condicionada y periódica de una transferencia monetaria directa a las familias en condición de pobreza y pobreza extrema. Este programa tiene como objetivo apoyar la culminación del ciclo de educación de los jóvenes pobres, contribuir a la prevención del embarazo a temprana edad o en la adolescencia, y promover factores de movilidad social entre las familias beneficiarías, en aras de estimular la superación de sus condiciones de pobreza. Estas leyes o acciones políticas implementadas por el gobierno nacional actual, se ven fortalecidas por el trabajo de organismos como la Agencia Nacional para la Superación de la Pobreza Extrema (ANSPE), creada a partir del Decreto 4160 de 2011 (Congreso de la República de Colombia, 2011 ); y las acciones de La Subdirección General para la Superación de la Pobreza de Prosperidad Social.

Ahora bien, no en el marco de las acciones para la superación de la pobreza, sino en el contexto legal que demarca la necesidad de hacer seguimiento a la situación de pobreza en el país, es preciso mencionar el Conpes Social 150 propuesto por el Departamento Nacional de Planeación (DNP, 2012). Este documento regula las metodologías oficiales y arreglos institucionales para la medición de la pobreza en Colombia, enfocándose en clarificar los instrumentos, técnicas de recolección de datos y responsables del cálculo monetario y multidimensional de este fenómeno social. Así, esta investigación se enmarca en el análisis aplicado de datos del IPM en los hogares colombianos, que publica el DANE, según lineamientos de esta normativa.

Pasando a referenciar las investigaciones sobre la pobreza en las que se utilizan datos y técnicas de modelación, a nivel latinoamericano es preciso mencionar los trabajos de Chacón-Mejía et al. (2021), Arias-Ramírez et al. (2020), Barahona (2018) y Fuentes et al. (2018). Los primeros autores, analizan las ruralidades en la región desde la perspectiva multidimensional de la pobreza, a partir de un Análisis de Componentes Principales y un Análisis de Correspondencia Canónica. Estos autores concluyen que, a pesar de que se observa heterogeneidad entre las múltiples dimensiones que determinan la pobreza, esta presenta homogeneidad al comparar entre países.

Arias-Ramírez et al. (2020) analizan la pobreza y desigualdad en Costa Rica, dando una mirada más allá de la distribución de los ingresos. Estos autores se enfocan en comparar las necesidades básicas insatisfechas (NBI) y las características espaciales de la pobreza, mediante análisis descriptivos, técnicas de georreferenciación y regresión ponderada. En esta investigación, se concluye: "El país necesita redefinir su estrategia de desarrollo para gestar un círculo virtuoso de crecimiento económico con mayores niveles de desarrollo humano" (p. 24).

Barahona (2018) compara indicadores de pobreza en México entre 2000 y 2010 , analizando correlaciones entre variables como desempleo, horas tv, asesinatos, muertes, consultas en biblioteca, oportunidades (programa de gobierno), cantidad de lugares históricos visitados, bienes y capacidades de la pobreza. Las técnicas de análisis usadas en esta investigación fueron: análisis de componentes principales (PCA), análisis factorial de mínimos cuadrados ponderados (WLS), y KMO (Kaiser-Meyer-Olkin), a través de las cuales se contrastó si las correlaciones parciales entre las variables eran lo suficientemente pequeñas para ser consideradas significativas. Gracias a esta investigación, el gobierno mexicano asignó recursos del programa Oportunidades a las provincias más pobres del país. 
Por su parte, Fuentes et al. (2018), analizando la medición multidimensional de la pobreza en México, usaron la metodología que desarrolló el Consejo Nacional de Evaluación de la Política de Desarrollo Social de México (CONEVAL) en 2010, para comparar la pobreza a nivel municipal. Con el fin de aplicar la metodología de Coneval a nivel intraurbano, se propuso como alternativa la construcción de unidades de análisis territorial, seleccionando muestras específicas para la aplicación de encuestas. En este estudio, se concluyó que la metodología a nivel intraurbano permite identificar a las personas que viven en la pobreza de forma más localizada, además de entender las privaciones sociales que más contribuyen a su condición de ser pobres.

Pasando al contexto de Colombia, es preciso referenciar a Mayorga-Henao et al. (2021), Marrugo et al. (2015) y Cardona et al. (2014). El primer autor estudia los patrones de distribución de la pobreza multidimensional en las principales aglomeraciones urbanas del país, mediante la aplicación de múltiples métodos de análisis espacial y estadísticos. En esta investigación, se concluye que la segregación de la pobreza en el país muestra una relación compleja entre variables espaciales y variables socioeconómicas, aunque a la distribución de la pobreza al interior de las ciudades cumple con el principio de autocorrelación espacial.

Marrugo et al. (2015), mediante un análisis descriptivo, se centra en el comportamiento de la pobreza en una región en específico: la Caribe. En esta investigación, se utilizan los datos de la Gran Encuesta Integrada de Hogares (GEIH), realizada por el DANE en el año 2012, usando como variable explicada la línea de pobreza (LP) delimitada a tres valores: 1) cuando el hogar es no pobre; 2 ) cuando el hogar es moderadamente pobre; y 3) cuando el hogar vive en condiciones de pobreza extrema. Posteriormente, se analiza el comportamiento de esta variable y su relación con características socioeconómicas de la población en la región Caribe. Los resultados mostraron que una menor educación y estar en condición de desempleo son los factores que más contribuyen a la probabilidad de ser pobre en la región (Marrugo et al., 2015).
Para terminar, Cardona et al. (2014) analizan mediante regresión lineal simple, datos del porcentaje de pobreza, pobreza extrema y el coeficiente de Gini (indicador de la desigualdad económica en una población), en los años 2010 y 2011 , para las trece principales ciudades de Colombia. Los autores realizaron una gráfica de dispersión donde se muestra que existe una relación entre la variable independiente (porcentaje de pobreza en 2010), y la variable dependiente (porcentaje de pobreza en 2011). Adicionalmente, se hallaron los coeficientes de regresión, intervalos de confianza, coeficientes de correlación y pruebas de significancia, para concluir que los valores observados y estimados están por debajo de la meta de pobreza nacional.

\section{Metodología}

Este estudio es de análisis cuantitativo y puede considerarse como descriptivo y exploratorio porque se busca analizar las relaciones existentes entre distintas variables de hogares y personas, identificando qué determina la condición de pobreza en Colombia, cuáles son los factores más preponderantes y aquellos que determinan las diferencias y similitudes por regiones. Para la obtención y explicación de los resultados, se utilizan análisis descriptivos, pruebas de hipótesis para diferencias de proporciones y la técnica multivariante PCA (análisis de componentes principales), interpretando los datos desde el comportamiento de variables individuales y desde asociaciones bivariantes y multidimensionales.

Las hipótesis en evaluación son dos. La primera: existen diferencias entre los niveles de pobreza de los hogares colombianos según características sociodemográficas de las personas que los componen; y la segunda: existen diferencias entre las condiciones de pobreza según regiones del país. En relación con los datos en análisis, es pertinente detallar las características o factores examinados desde la base de datos IPM (DANE, 2019); la cual está disponible de manera gratuita en el Banco Nacional de Datos de la web de microdatos de la 
institución, teniendo disponibilidad de información a nivel nacional y departamental. En la Tabla 1, se describe el diccionario de variables de interés.

La base de datos del IPM (DANE, 2019) cuenta con una muestra de 88.713 hogares, que representan 283.012 personas a nivel nacional y que proyectan el total de la población del país.
El detalle de la distribución de esta muestra se ilustra en la Tabla 2, donde se incluyen los valores absolutos del total de hogares y las personas que viven en ellos, según su condición de ser pobres o no, haciendo distinciones por regiones. Se puede observar que es mayor la cantidad de hogares no pobres, frente a los pobres, y que la región con mayor cantidad de hogares pobres es la Caribe.

Tabla 1. Variables analizadas

\begin{tabular}{|c|c|c|}
\hline Notación & Tipo & Significado y Categorías \\
\hline REG & Localización & $\begin{array}{l}\text { Región: } 1 \text { Caribe, } 2 \text { Oriental, } 3 \text { Central, } 4 \text { Pacífica (sin Valle), } 5 \text { Bogotá, } 6 \text { Antioquia, } 7 \text { Valle } \\
\text { del Cauca, } 8 \text { San Andrés y Providencia, } 9 \text { Orinoquía - Amazonía }\end{array}$ \\
\hline $\mathrm{POB}$ & Hogar & Hogar pobre y no pobre \\
\hline PAR & Hogar & Privación o no por inadecuado material de paredes exteriores \\
\hline PIS & Hogar & Privación o no por inadecuado material de pisos \\
\hline ALC & Hogar & Privación o no por inadecuada eliminación de excretas \\
\hline $\mathrm{ACU}$ & Hogar & Privación o no por no acceso a fuente de agua mejorada \\
\hline EMF & Hogar & Privación o no por Tasa de Empleo Formal \\
\hline DLD & Hogar & Privación o no por Desempleo de Larga Duración \\
\hline BAS & Hogar & Privación o no por barreras de acceso a salud \\
\hline ASS & Hogar & Privación o no por no aseguramiento en salud \\
\hline TRI & Hogar & Privación o no por Trabajo Infantil \\
\hline ATI & Hogar & Privación o no por Atención Integral a la Primera Infancia \\
\hline INE & Hogar & Privación o no por Inasistencia Escolar \\
\hline RES & Hogar & Privación o no por rezago escolar \\
\hline ANA & Hogar & Privación o no por Analfabetismo \\
\hline LOE & Hogar & Privación o no por Bajo Logro Educativo \\
\hline HAC & Hogar & Privación o no por hacinamiento crítico \\
\hline CUA & Hogar & Número de cuartos en los que duermen las personas del hogar \\
\hline PER & Hogar & Número de personas que componen el hogar \\
\hline IPM & Hogar & Índice de Pobreza Multidimensional \\
\hline PRI & Personas & Número de personas en el hogar que tienen como máximo un nivel educativo de primaria \\
\hline SEC & Personas & Número de personas que tienen como máximo un nivel educativo de secundaria \\
\hline SUP & Personas & Número de personas que tienen como máximo un nivel educativo de Educación Superior \\
\hline NDE & Personas & Número de personas del hogar que no declaran su formación académica \\
\hline MEN & Personas & Número de personas en el hogar menores a 18 años de edad \\
\hline ADJ & Personas & Número de personas en el hogar con edad de 18 a 29 años \\
\hline ADT & Personas & Número de personas en el hogar con edad de 30 a 54 años \\
\hline MAY & Personas & Número de personas en el hogar mayores o iguales a 55 años \\
\hline $\mathrm{HOM}$ & Personas & Número de personas en el hogar de género masculino \\
\hline MUJ & Personas & Número de personas en el hogar de género femenino \\
\hline
\end{tabular}


Sociedad y Economía N 44 (Sep - dic 2021) / e-ISSN: 2389-9050 / e10310734

Edith Johana Medina-Hernández, Luis Francisco Sierra-lbáñez y Andy Rafael Domínguez-Monterrosa

Tabla 2. Hogares y personas analizadas según condición de ser pobres y no

\begin{tabular}{|c|c|c|c|c|c|c|}
\hline \multirow[b]{2}{*}{ Región } & \multicolumn{3}{|c|}{ Total de hogares analizados } & \multicolumn{3}{|c|}{ Personas en los hogares analizado } \\
\hline & No pobre & Pobre & Total & No pobre & Pobre & Total \\
\hline Caribe & 13.215 & 6.827 & 20.042 & 45.070 & 28.739 & 73.809 \\
\hline Oriental & 13.277 & 2.920 & 16.197 & 39.314 & 10.117 & 49.431 \\
\hline Central & 15.259 & 3.418 & 18.677 & 43.453 & 11.978 & 55.431 \\
\hline $\begin{array}{l}\text { Pacífica } \\
\text { (sin Valle) }\end{array}$ & 5.965 & 2.526 & 8.491 & 18.101 & 9.161 & 27.262 \\
\hline Bogotá & 1.688 & 51 & 1.739 & 4.707 & 177 & 4.884 \\
\hline Antioquia & 2.767 & 650 & 3.417 & 8.038 & 2.330 & 10.368 \\
\hline Valle del Cauca & 2.683 & 429 & 3.112 & 7.854 & 1.568 & 9.422 \\
\hline San Andrés y Providencia & 1.161 & 87 & 1.248 & 2.735 & 230 & 2.965 \\
\hline Orinoquía y Amazonía & 11.160 & 4.630 & 15.790 & 32.597 & 16.843 & 49.440 \\
\hline Total nacional & 67.175 & 21.538 & 88.713 & 201.869 & 81.143 & 283.012 \\
\hline
\end{tabular}

\subsection{Técnicas para el análisis de la información}

A continuación se presentan los conceptos técnicos relacionados con la obtención de los resultados. En primera instancia, se definen aspectos relacionados con la construcción del indicador IPM por parte del DANE y, posteriormente, se incluyen conceptos relativos al método multivariante PCA, que son necesarios para la comprensión de los hallazgos del trabajo.

El cálculo del IPM, en Colombia, se basa en la definición de variables de privación y sus ponderaciones. Desde estas se determina si un hogar se encuentra en condiciones de pobreza o no. Para esto, según como se especifica en DANE (2018), se utiliza un algoritmo que establece como unidad de análisis los hogares y una equiponderación de cinco dimensiones y los factores que las definen. En este algoritmo se procede a construir una matriz de 1 o 0 , donde el 1 representa privación y 0 , no privación. En la matriz, las filas representan los hogares y las columnas las características evaluadas, las cuales fueron incluidas en la Tabla 1.

Dorian (2017) explica como las ponderaciones asignadas a cada uno de los factores de privación de los hogares se basan en el método desarrollado por Sabina Alkire y James Foster, en el marco del proyecto OPHI (Oxford Poverty \& Human Development Initiative), basado en las medidas de pobreza de Foster- GreerThorbecke. El método consiste en contar los diferentes tipos de privación que las personas experimentan al mismo tiempo y una vez establecidas las ponderaciones deseadas para cada factor, se realiza una suma ponderada para cada hogar, denominada indicador IPM. En Colombia, los hogares son considerados pobres multidimensionalmente cuando tienen privación en por lo menos el $33 \%$ de los indicadores (DANE, 2018).

Ahora bien, en cuanto a la técnica específica mediante la cual se obtienen los resultados en este estudio, el Análisis de Componentes Principales (PCA), es necesario indicar que es una técnica estadística planteada por Hotelling (1933), quien se basó en los trabajos de Pearson (1901) acerca de los ajustes ortogonales por mínimos cuadrados. El PCA es una técnica multivariante que realiza una reducción de dimensionalidad, creando un conjunto de datos menor al conjunto de variables originales, al cual se le denomina componentes principales y se expresa como combinaciones lineales de las variables originales.

Para Peña (2002), las componentes principales tienen doble utilidad. La primera es que permiten representar óptimamente los datos 
analizados en un espacio de dimensión pequeña, y la segunda, que permiten transformar las variables originales, que están correlacionadas entre sí, en nuevas variables incorrelacionadas, facilitando la interpretación de los datos. Así pues, con esta técnica lo que se busca es tener la mejor representación de los atributos de la información en análisis, en la menor cantidad de dimensiones posible.

En el PCA se dispone de una matriz $V$, que contiene una muestra de tamaño $\mathrm{n}$ acerca de $\mathrm{p}$ variables $X_{1}, X_{2}, \ldots, X_{p}$ inicialmente correlacionadas; para, posteriormente, obtener a partir de ellas un número $\mathrm{k} \leq \mathrm{p}$ de variables incorrelacionadas $C_{1}, C_{2}, \ldots, C_{p}$ que sean combinación lineal de las variables iniciales y que expliquen la mayor parte de su variabilidad.

La primera componente principal, al igual que las restantes, se expresa como combinación lineal de las variables originales. $\mathrm{C}_{1}$ se obtiene de forma que su varianza sea máxima, sujeta a la restricción de que la suma de los pesos $u_{1 j}$ al cuadrado sea igual a la unidad, es decir, la variable de los pesos o ponderaciones $\left(u_{11}, u_{12}, \ldots, u_{1 p}\right)$ se toma normalizada. Se trata entonces de hallar $C_{1}$ maximizando $V\left(C_{1}\right)=u_{1} V u_{1}$, sujeta a la restricción:

$$
\sum_{j=1}^{p} u_{l i}^{2}=u_{1} u_{1}=1
$$

Se demuestra que, para maximizar $V\left(C_{1}\right)$ se toma el mayor valor propio $\lambda$ de la matriz $V$. Sea $\lambda_{1}$ el mayor valor propio de $\mathrm{V} y$ tomando $u_{1}$ como su vector propio normalizado $\left(u_{1} u_{1}=1\right)$. Teniendo definido el vector de ponderaciones que se aplica a las variables iniciales para obtener la primera componente principal, que está definida como:

$$
C_{1}=u_{1} X=u_{11} X_{1}+u_{12} X_{2}+\cdots+u_{1 p} X_{p}
$$

Para maximizar $V\left(C_{2}\right)$ se toma el segundo mayor valor propio $\lambda$ de la matriz $\mathrm{V}$ (el mayor ya se había tomado al obtener la primera componente principal). Tomando $\lambda_{2}$ como el segundo valor propio de $V$ y tomando $u_{2}$ como su vector propio asociado normalizado $\left(u_{2} u_{2}=1\right)$, ya se tendría definido el vector de ponderaciones que se aplica a las variables iniciales para obtener la segunda componente principal, definida como:

$$
C_{2}=u_{2} X=u_{21} X_{1}+u_{22} X_{2}+\cdots+u_{2 p} X_{p}
$$

De forma similar, la componente principal h-ésima se define como $C_{h}=X u_{h}$ donde $u_{h}$ es el vector propio de $\mathrm{V}$ asociado a su h-ésimo mayor valor propio. Cuanto mayor sea la varianza, mayor es la información que lleva incorporada la respectiva componente. Por esta razón, se selecciona como primera componente aquella que tiene mayor varianza, mientras que, por el contrario, la última es la de menor varianza.

Entre los distintos métodos multivariantes, los cuales, según Cuadras (2014), son un conjunto de técnicas estadísticas destinadas a describir e interpretar varias variables en conjunto, el PCA es considerado un método de interdependencia, porque permite encontrar asociaciones múltiples entre las variables, sin necesidad de examinar una variable respuesta en particular. Habitualmente los resultados del PCA y otras técnicas de reducción de la dimensionalidad suelen representarse mediante gráficos Biplot, propuestos por Galindo (1986), los cuales muestran conjuntamente las observaciones (filas de la matriz de datos) en forma de puntos, y las variables (columnas) en forma de vectores.

\section{Resultados}

Antes de presentar los resultados de la investigación obtenidos mediante la aplicación del PCA, es pertinente presentar una exploración descriptiva de las principales variables en estudio, haciendo comparaciones por regiones. Todo ello con la intención de discutir posibles acciones requeridas para la mitigación del fenómeno de la pobreza en el país, tanto en el ámbito nacional, como regional. Todas las figuras presentadas en este aparte del documento fueron obtenidas en el programa estadístico $\mathrm{R}$.

\subsection{Exploración descriptiva de los datos}

La primera gráfica por describir es la que representa las proporciones de hogares pobres y no pobres según regiones, de acuerdo con 
los totales que se presentaron anteriormente en la Tabla 2 y que se representan en la Figura 1. Puede observarse que la región más pobre es la Caribe, con un 34\% de hogares pobres, y que la segunda región con mayor prevalencia de hogares pobres es la Pacífica, con un 30\%, seguida de la Orinoquía y la Amazonía, 29\%. Estas diferencias observadas se probaron mediante un test chi cuadrado para igualdad de proporciones y se obtuvo que son estadísticamente significativas con un nivel de confianza del 99\% (valor $p<0,01$ ).

En la Figura 2, se muestra por regiones el porcentaje de hogares que tienen privación y no privación de logro educativo (LOE). Se observa que la región con mayor privación por logro educativo es la región Pacífica: $62 \%$ frente al $38 \%$ de no privación; es decir, esta región tiene un alto porcentaje de hogares que no tienen logro educativo alguno. La segunda región con mayor porcentaje de privación LOE, es la central, con un $61 \%$, y la tercera la Oriental, con $60 \%$. Desde esta gráfica, también se interpreta que siete de las nueve regiones estudiadas tienen un porcentaje mayor del $50 \%$ en privación por LOE.

Las diferencias percibidas en la Figura 2, se probaron estadísticamente y se obtuvo valor $p$ $<0,05$, entonces se concluye que, con una confianza del $95 \%$, sí existe una diferencia entre la proporción de privación por logro educativo en los hogares colombianos según regiones.

En la Figura 3, se presenta la gráfica de los hogares según proporciones de acceso, o no, a un empleo formal. Se observan a Bogotá y San Andrés y Providencia como las regiones donde menor privación en la variable EMF se registra en el país. En contraste las regiones donde más del $85 \%$ de los hogares declaran tener dificultades de acceso a condiciones dignas de empleabilidad, entre al menos uno de los integrantes de la familia, son el Caribe colombiano, el Pacífico y la Amazonía-Orinoquía. Las diferencias porcentuales percibidas en la Figura 3 fueron probadas mediante un test para diferencia de proporciones y son estadísticamente significativas con una confianza del 99\% (valor $\mathrm{p}<0,01$ ).
Además de los factores de privación que incluye el DANE en la definición del indicador IPM, las características sociodemográficas de las personas que componen los hogares, también permiten concluir frente a otros atributos que caracterizan el fenómeno de la pobreza. Por ello, a continuación se ejemplifica el comportamiento del número de personas con educación superior, para advertir diferencias entre regiones.

En la Figura 4, se observa cómo las regiones con mayor porcentaje de personas sin estudios superiores son la Pacífica y la Orinoquía y Amazonía (76,9\%). Por su parte, la región con el menor porcentaje de hogares donde no existe ni una persona que tenga estudios superiores es Bogotá (38,2\% de los hogares). En general, siete de las nueve regiones analizadas muestran cómo más de un $70 \%$ de las personas no tiene educación superior.

\subsection{Resultados del análisis mediante PCA}

Antes de presentar los resultados de las componentes obtenidas, conviene explicar la estructura de correlaciones observadas entre pares de variables, según las representaciones de las matrices de correlaciones en la Figura 5 y Figura 6. Desde estas, es posible notar cómo los distintos factores en el análisis tienen correlaciones positivas o negativas con el indicador IPM; por ejemplo, desde la Figura 5 se interpreta que las mayores correlaciones las presenta el IPM con el logro educativo, el empleo formal y el analfabetismo (LOE: 0,68 , EMF: 0,58 y ANA: 0,53 ); en contraste, las correlaciones más bajas se registran con la atención integral a la primera infancia y el trabajo infantil (ATI: 0,13 y TRI: 0,18).

Las correlaciones que se observan entre las variables de personas, en la Figura 6, son más altas que las vistas para las variables de privación de los hogares, en la Figura 5. Este resultado en términos interpretativos, justifica el porqué este estudio aporta al análisis de la pobreza como fenómeno multivariante; porque si bien el IPM es un índice multidimensional desde el cual se hacen oficialmente las clasificaciones de hogares pobres y no pobres en 
Figura 1. Porcentaje de Hogares pobres y no pobres por regiones de Colombia

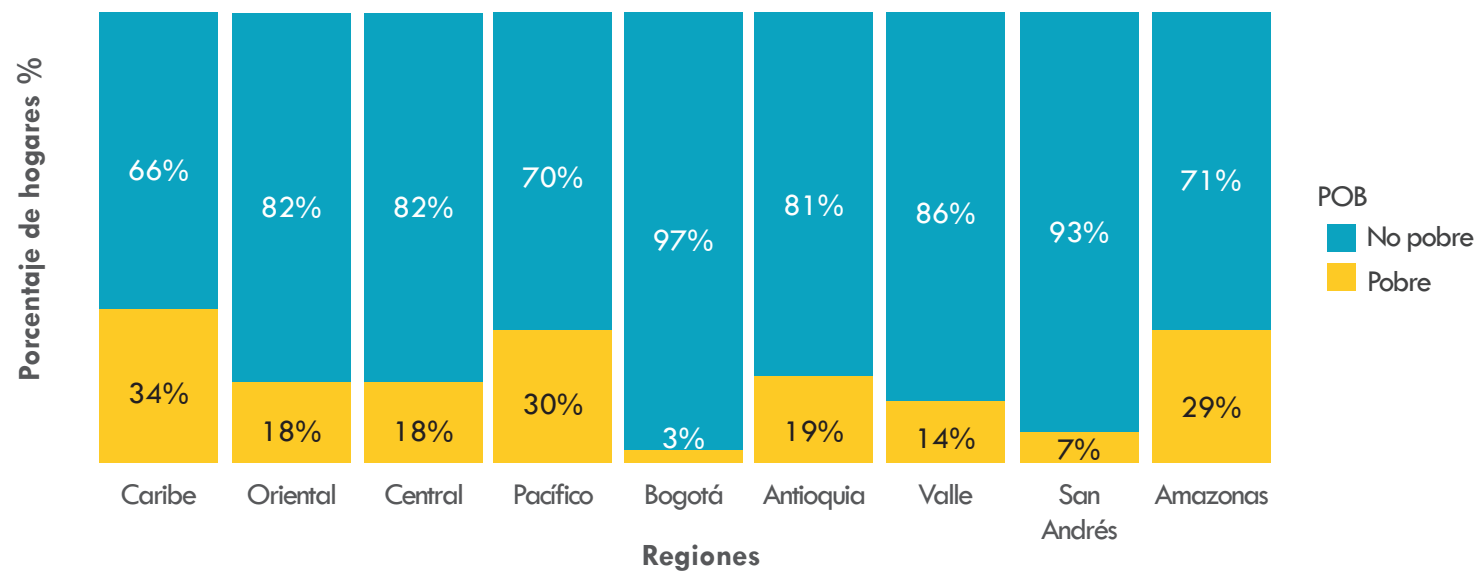

Fuente: elaboración propia en el programa estadístico $R$, con datos IPM (DANE, 2019).

Figura 2. Privación por logro educativo según regiones de Colombia

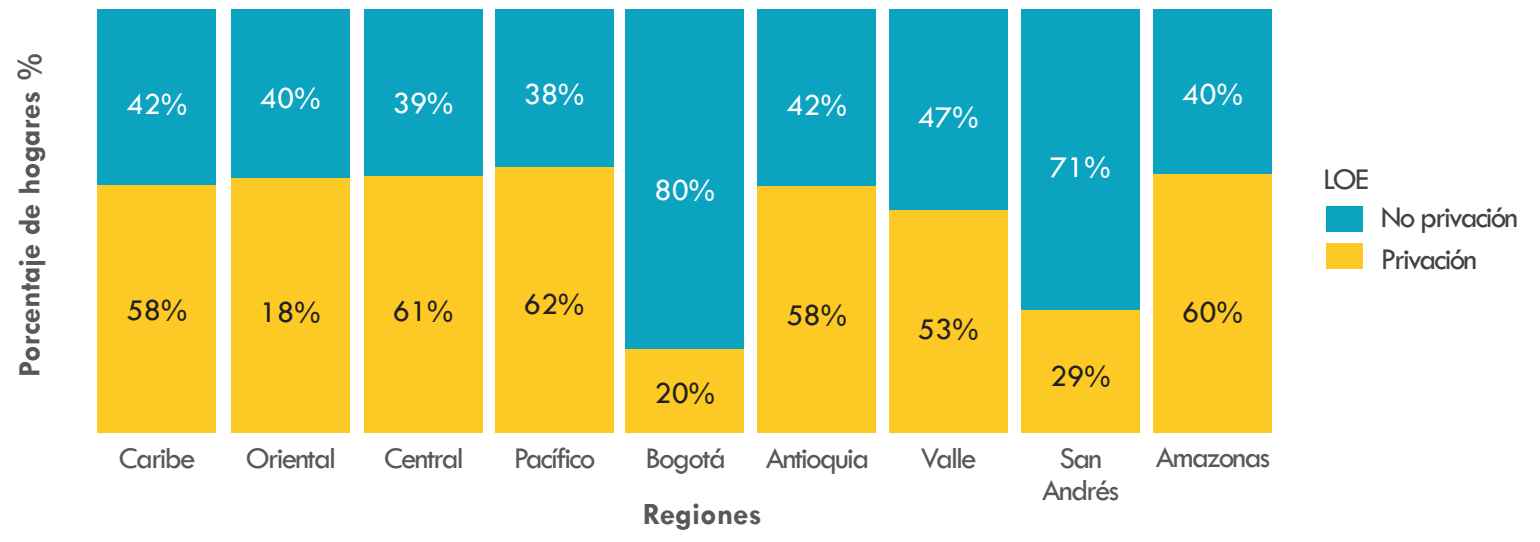

Fuente: elaboración propia en programa estadístico R, con datos IPM (DANE, 2019).

Figura 3. Privación por condiciones de empleo formal según regiones

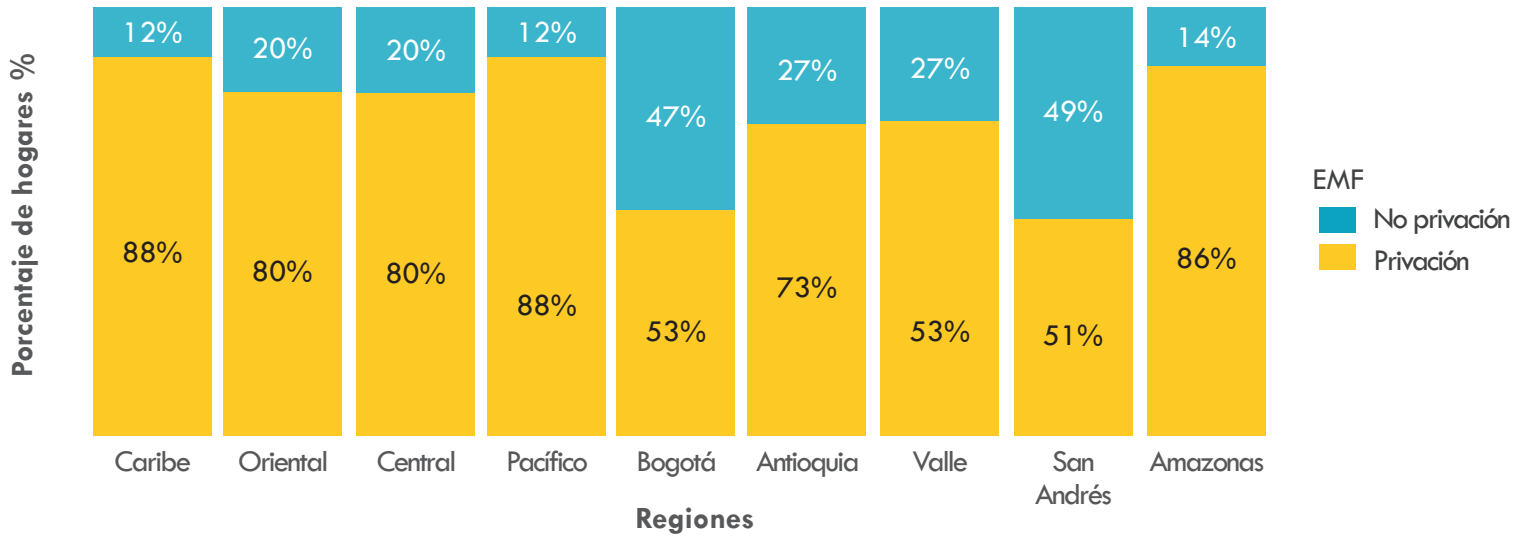

Fuente: elaboración propia en programa estadístico R, con datos IPM (DANE, 2019). 
Figura 4. Porcentaje de personas en el hogar con educación superior por regiones

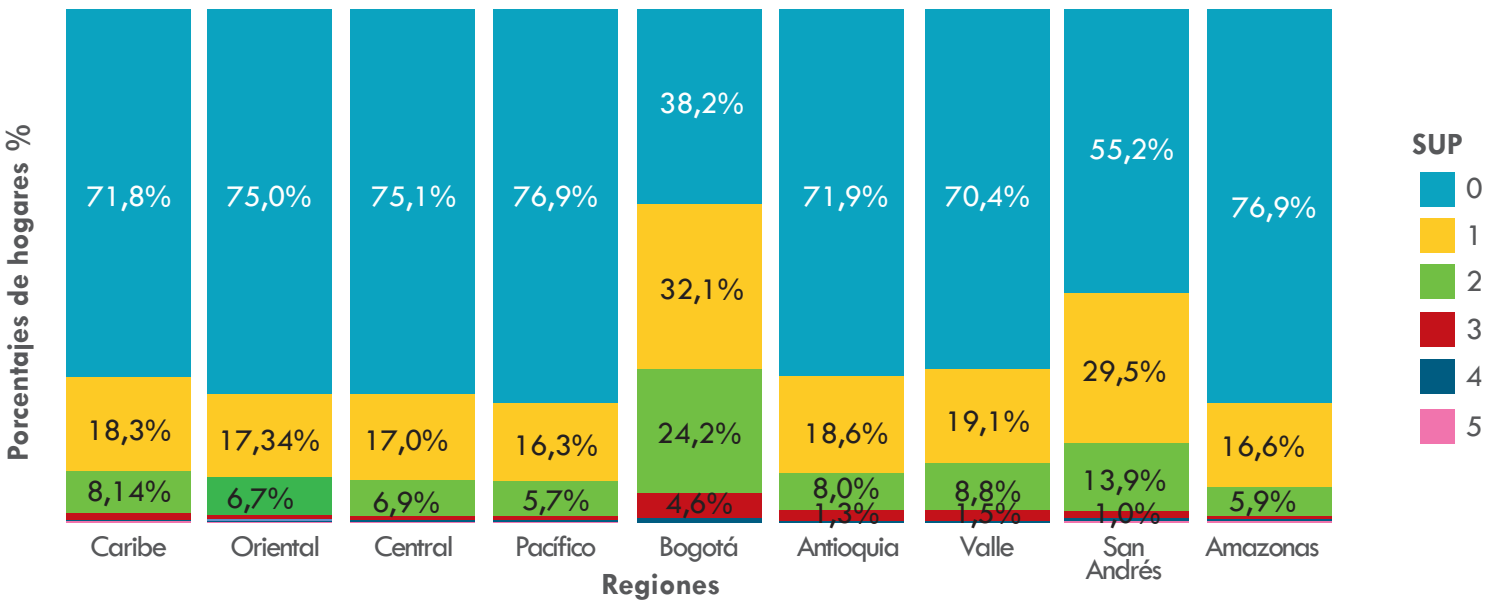

Fuente: elaboración propia en programa estadístico $R$, con datos IPM (DANE, 2019).

Figura 5. Matriz de correlaciones bivariadas entre las variables de Hogares y el IPM

\begin{tabular}{|c|c|c|c|c|c|c|c|c|c|c|c|c|c|c|c|c|c|}
\hline PAR & 0 & 0 & & & & & & & & $\theta$ & & & & 0 & & & 0 \\
\hline 0,23 & PIS & 0 & 0 & & & & & & & 0 & & 0 & 0 & 0 & 0 & & \\
\hline 0,24 & 0,31 & ALC & 0 & & & & & & & 0 & & (1) & 0 & 0 & & & \\
\hline 0,09 & 0,22 & 0,35 & ACU & & & & & & & 0 & & (1) & 0 & & & & \\
\hline 0,06 & 0,14 & 0,11 & 0,14 & EMF & $\theta$ & & & & & 0 & & (1) & 0 & & & & \\
\hline 0,03 & 0,03 & 0,02 & 0,01 & 0,06 & DLD & & & & & 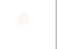 & & $\circ$ & & & 0 & 0 & \\
\hline 0,00 & 0,00 & 0,01 & $-0,01$ & 0,04 & $-0,04$ & BAS & & & & & & & & & & & \\
\hline 0,02 & 0,03 & 0,03 & 0,02 & 0,13 & $-0,06$ & 0,05 & ASS & & & (1) & & & & & & & \\
\hline 0,01 & 0,03 & 0,03 & 0,03 & 0,05 & $-0,06$ & 0,04 & 0,03 & TRI & & 0 & O & & & & & - & \\
\hline 0,03 & 0,02 & 0,04 & 0,02 & 0,00 & $-0,05$ & 0,02 & 0,12 & 0,01 & ATI & $\theta$ & & & & 0 & & 0 & \\
\hline 0,05 & 0,07 & 0,07 & 0,06 & 0,07 & $-0,04$ & 0,03 & 0,10 & 0,28 & 0,07 & INE & 0 & $\theta$ & & 0 & & 0 & \\
\hline 0,06 & 0,07 & 0,07 & 0,06 & 0,08 & $-0,10$ & 0,06 & 0,03 & 0,21 & 0,05 & 0,25 & RES & & & 0 & 0 & 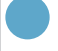 & \\
\hline 0,05 & 0,19 & 0,15 & 0,13 & 0,15 & 0,06 & 0,02 & 0,04 & 0,04 & $-0,03$ & 0,06 & 0,03 & ANA & 0 & & & & \\
\hline 0,06 & 0,20 & 0,15 & 0,23 & 0,33 & 0,07 & 0,01 & 0,02 & 0,07 & $-0,06$ & 0,10 & 0,11 & 0,34 & LOE & & & & \\
\hline 0,14 & 0,15 & 0,14 & 0,04 & 0,07 & $-0,05$ & 0,05 & 0,08 & 0,04 & 0,18 & 0,13 & 0,18 & 0,04 & 0,04 & HAC & (1) & 0 & 0 \\
\hline$-0,03$ & $-0,07$ & $-0,02$ & $-0,02$ & 0,00 & $-0,08$ & 0,02 & 0,01 & 0,03 & 0,05 & 0,03 & 0,14 & 0,02 & $-0,02$ & $-0,10$ & CUA & 0 & \\
\hline 0,05 & 0,05 & 0,05 & 0,01 & 0,11 & $-0,22$ & 0,10 & 0,10 & 0,14 & 0,25 & 0,21 & 0,50 & 0,08 & 0,05 & 0,34 & 0,30 & PER & \\
\hline 0,22 & 0,39 & 0,38 & 0,38 & 0,58 & 0,26 & 0,23 & 0,32 & 0,18 & 0,13 & 0,27 & 0,31 & 0,53 & 0,68 & 0,25 & $-0,01$ & 0,23 & IPM \\
\hline
\end{tabular}


Figura 6. Matriz de correlaciones bivariadas entre las variables de personas y el IPM

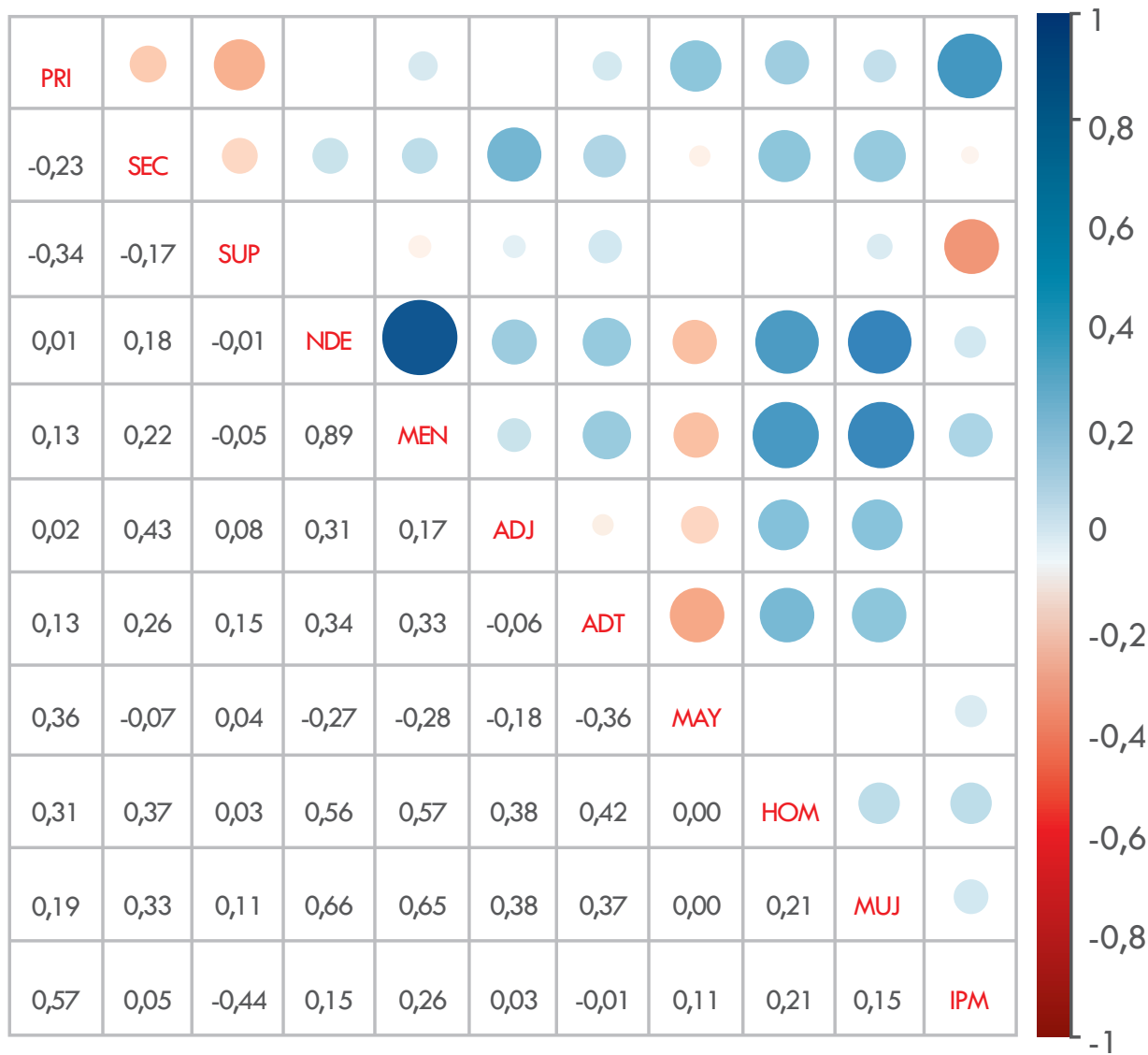

Fuente: elaboración propia en programa estadístico $R$, con datos IPM (DANE, 2019).

Colombia, con el método Alkire y Foster que se usa en DANE (2018) para asignar las ponderaciones de los factores de privación, no se considera ninguna dependencia frente al número de personas de los hogares ni respecto a las características que definen a tales personas, aunque estas también aportan al análisis multidimensional de la pobreza.

Continuando con el análisis y pasando a los resultados específicos de la aplicación del PCA, es pertinente indicar el porcentaje de varianza explicada por cada una de las componentes principales obtenidas. Los dos primeros ejes resumen el $32,7 \%$ de la variabilidad en los datos y se observa que hasta la dimensión 4 se logra explicar $44,2 \%$, por lo cual, con analizar los planos 1-2 y 3-4 se podría lograr entender qué es lo que caracteriza a los hogares del país, según sus condiciones de pobreza.
Para entender los resultados de la aplicación de la técnica y antes de presentar de forma gráfica los principales planos del PCA, es necesario analizar las ponderaciones obtenidas para las variables en análisis. En la Tabla 3, se muestran los coeficientes obtenidos para las cinco primeras componentes principales.

Desde la Tabla 3 se puede concluir que, al remplazar los valores obtenidos en la ecuación (2), presentada anteriormente en la sección de metodología, la ecuación de la primera componente es:

$$
\begin{gathered}
C_{1}=u_{1} X=u_{11} X_{1}+u_{12} X_{2}+\cdots+u_{1 p} X_{p}= \\
C_{1}=0,37 \mathrm{PAR}+0,66 \mathrm{PIS}+\cdots+15,55 \mathrm{PER}+\cdots+ \\
13,12 \mathrm{MEN}+\cdots+9,28 \mathrm{MUJ}
\end{gathered}
$$


Frente a las mayores contribuciones de la componente 1 , se exaltan las ponderaciones obtenidas para las variables: \# de personas en el hogar (PER), \# de menores en el hogar (MEN) y \# de personas en el hogar que no declaran su nivel educativo (NDE). En relación con la componente principal 2 , se obtiene que las mayores ponderaciones se concentran sobre las variables (IPM), logro educativo (LOE), \# de personas en el hogar con primaria, alfabetismo (ANF) y \# de personas en el hogar con secundaria (SEC).

$$
\begin{gathered}
C_{2}=u_{2} X=u_{21} X_{1}+u_{22} X_{2}+\cdots+u_{2 p} X_{p}= \\
C_{2}=0,91 \text { PAR }+4,28 \mathrm{PIS}+\cdots+15,79 \text { LOE }+\cdots+1,47 \mathrm{MUJ}
\end{gathered}
$$

Pasando a las representaciones de las componentes, en la Figura 7, se grafican los hogares y las variables de privación en el mismo plano, asignando por colores la condición de ser pobre o no. Se observa que los vectores más representativos, por ser los de mayor variabilidad (los más largos), corresponden a las variables: número de personas en el hogar (PER), número de menores en el hogar (MEN), núme-

\begin{tabular}{|c|c|c|c|c|c|}
\hline Variable & $\operatorname{Dim}, 1$ & $\operatorname{Dim}, 2$ & $\operatorname{Dim}, 3$ & $\operatorname{Dim}, 4$ & $\operatorname{Dim}, 5$ \\
\hline PAR & 0,37 & 0,91 & 9,31 & 0,15 & 2,91 \\
\hline PIS & 0,66 & 4,28 & 8,32 & 0,04 & 2,22 \\
\hline ALC & 0,69 & 3,45 & 12,88 & 0,04 & 2,13 \\
\hline $\mathrm{ACU}$ & 0,38 & 4,48 & 6,1 & 0,53 & 0 \\
\hline EMF & 1,13 & 5,83 & 0,01 & 6,18 & 1,62 \\
\hline DLD & 0,8 & 2,2 & 0,09 & 0,1 & 6,87 \\
\hline BAS & 0,37 & 0,06 & 0,04 & 1,24 & 0,16 \\
\hline ASS & 0,54 & 0,26 & 0,14 & 13,37 & 2,38 \\
\hline TRI & 0,96 & 0,19 & 0,06 & 0,25 & 17,07 \\
\hline ATI & 1,74 & 0,33 & 1,26 & 1,42 & 3,83 \\
\hline INE & 1,96 & 0,62 & 0 & 0,07 & 12,78 \\
\hline RES & 7,32 & 0,02 & 0,13 & 8,26 & 2,24 \\
\hline ANA & 0,44 & 8,17 & 3,64 & 0,13 & 3,24 \\
\hline LOE & 0,77 & 15,79 & 2,43 & 0,78 & 0,86 \\
\hline HAC & 3,67 & 0,01 & 5,28 & 0,86 & 2,62 \\
\hline CUA & 1,33 & 0,73 & 7,79 & 0,03 & 0,41 \\
\hline PER & 15,55 & 1,27 & 2,77 & 0,12 & 1,39 \\
\hline IPM & 4,23 & 17,63 & 0,38 & 0,91 & 0 \\
\hline PRI & 2,48 & 11,19 & 11,34 & 1,29 & 0,02 \\
\hline SEC & 2,75 & 2,75 & 0,01 & 22,56 & 4,66 \\
\hline SUP & 0,25 & 8,49 & 0,72 & 0,88 & 7,77 \\
\hline NDE & 11,68 & 2,19 & 0,85 & 2,79 & 2,83 \\
\hline MEN & 13,12 & 0,75 & 1,17 & 4 & 0,53 \\
\hline ADJ & 3,28 & 1,56 & 0 & 24,97 & 0,56 \\
\hline ADT & 4,11 & 2,16 & 0,39 & 7,28 & 5,53 \\
\hline MAY & 0,52 & 2,94 & 21,51 & 1,5 & 10,69 \\
\hline HOM & 9,61 & 0,28 & 1,94 & 0,25 & 0,12 \\
\hline MUJ & 9,28 & 1,47 & 1,45 & 0 & 4,56 \\
\hline
\end{tabular}

Tabla 3. Coeficientes de las cinco primeras componentes principales 
Figura 7. Plano 1-2 de componentes principales según hogares pobres o no

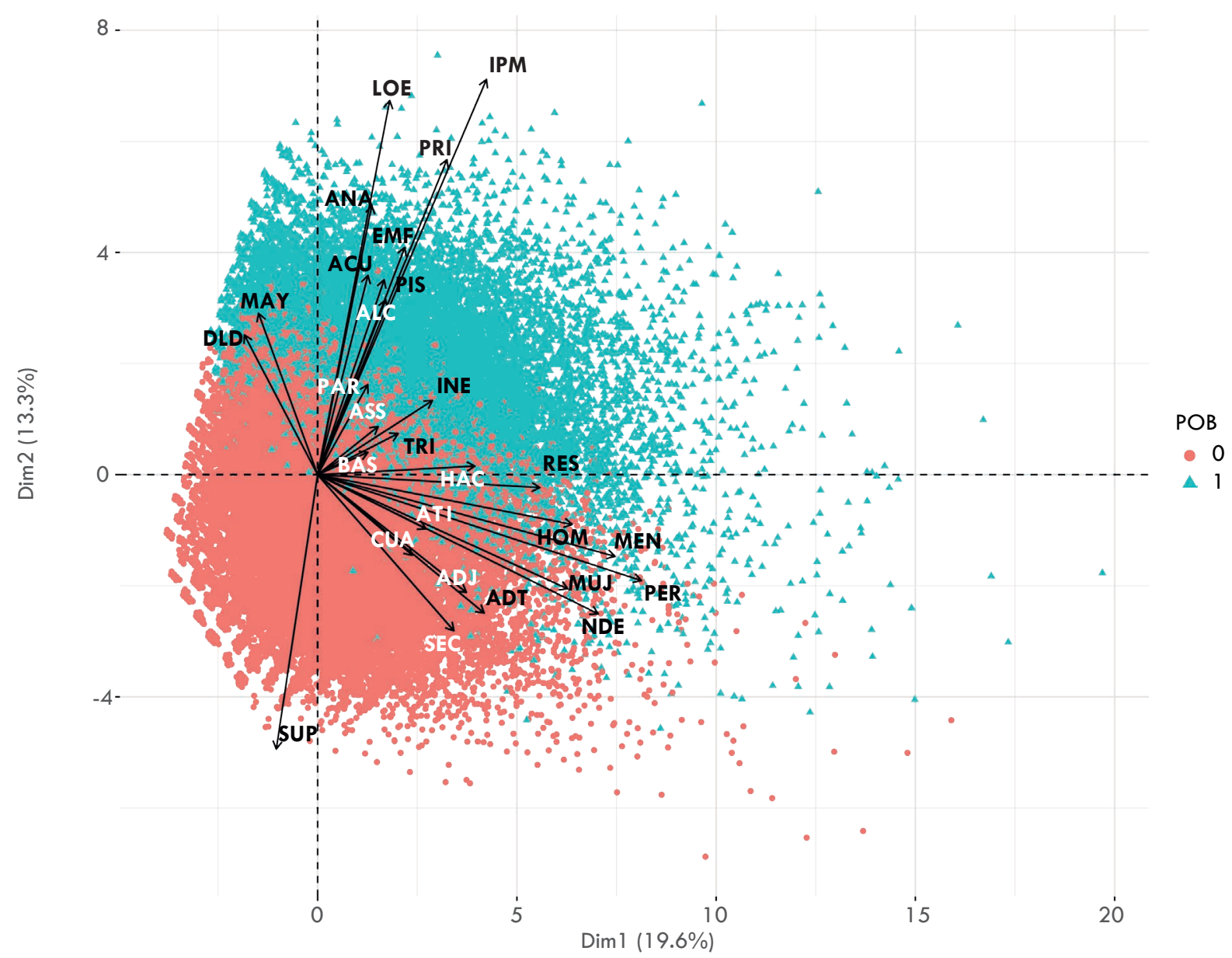

Fuente: elaboración propia en programa estadístico R, a partir de datos IPM (DANE, 2019).

ro de personas con primaria (PRI) y número de personas con educación superior (SUP).

Las variables de privación que van en la dirección del vector del IPM, que se observan en alta correlación con este, por tener ángulos pequeños frente a él y a su vez mostrarse como vectores largos en el plano, son las condiciones de privación por logro educativo (LOE), empleo formal (EMF) y el vector de analfabetismo (ANA). En dirección contraria, se observa la educación superior (SUP) caracterizando a los hogares que no son pobres.

En la Figura 8, donde se muestra plano 1-2 del Biplot, asignando por colores el número de personas que viven en cada hogar, se observa claramente un patrón de ordenación en el sen- tido de la primera componente principal, de forma que los hogares con mayor cantidad de personas se ubican hacia la derecha. Entre tales hogares, también se notan muchos menores de edad que están realizando o cursando la secundaria. En contraste, los hogares donde residen pocas personas, ubicados hacia la izquierda del plano, se caracterizan por tener integrantes mayores de edad, y personas con periodos de desempleo de larga duración.

Ahora bien, en el análisis de las componentes principales no solo es importante interpretar el plano 1-2, dado que otros planos y dimensiones también reflejan patrones del comportamiento de los datos. En la Figura 9, se presenta el plano de las componentes principales 3 y 4 , pintando el número de personas mayores 
Figura 8. Biplot en el plano 1-2 según número de personas en el hogar

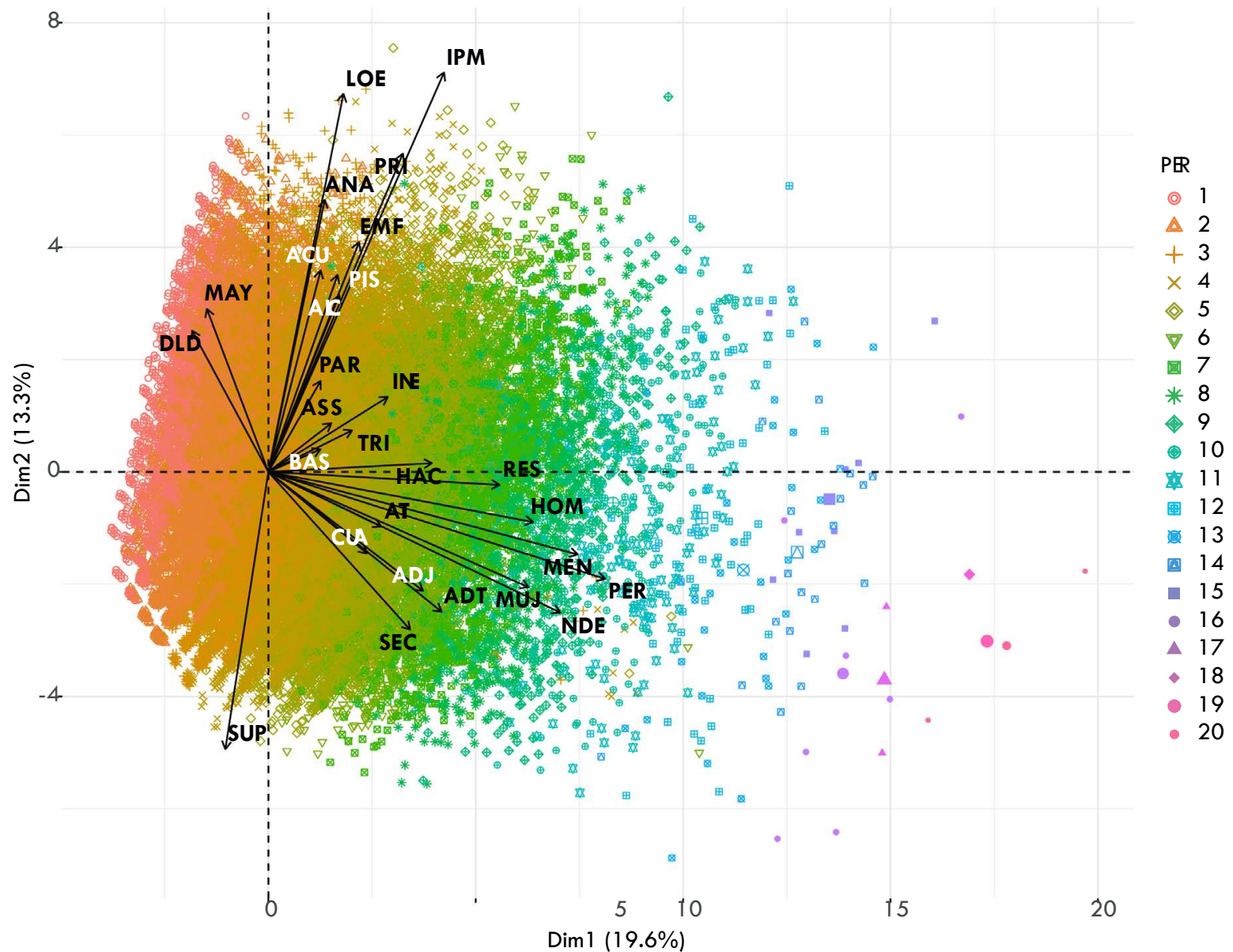

Fuente: elaboración propia en programa estadístico $R$, a partir de datos IPM (DANE, 2019).

que viven en los hogares; se observa claramente la diferenciación en la dirección del eje 3. Frente al eje 4 puede indicarse que los adultos jóvenes (ADJ) se notan caracterizados por tener empleo formal (EMF) y aseguramiento en salud (ASS).

\section{Discusión de resultados}

De acuerdo con los resultados presentados anteriormente, resulta útil discutir sus implicaciones y utilidad desde el punto de vista interpretativo. El primero de los hallazgos que vale la pena evaluar es que el factor obtenido como el más importante para caracterizar la pobreza de los hogares colombianos, es el logro educativo. Esta idea la sustenta la Organización para la Cooperación y el Desarrollo Económico (OCDE, 2016), cuando, analizando históricamente la evo- lución del acceso a la educación en Colombia, afirma que "muchos obstáculos limitan el acceso de los jóvenes a la educación, entre otros, la falta de oportunidades educativas, la pobreza, la presión por empezar a trabajar, el conflicto y la violencia" (p. 30).

Este primer hallazgo puede deberse a que los niños, jóvenes o adultos jóvenes en lugar de estudiar, muchas veces deben asumir de forma temprana actividades laborales, por causa de limitantes económicos en sus hogares. Por ello, sería necesario reevaluar estrategias con el fin de disminuir la incidencia de este factor en el cálculo del IPM, para que no sea visto simplemente como un ponderador de un indicador multidimensional de la pobreza, sino como un condicionante del derecho a la educación de los menores de edad. 
Figura 9. Biplot en el plano 3-4 según personas mayores en el hogar

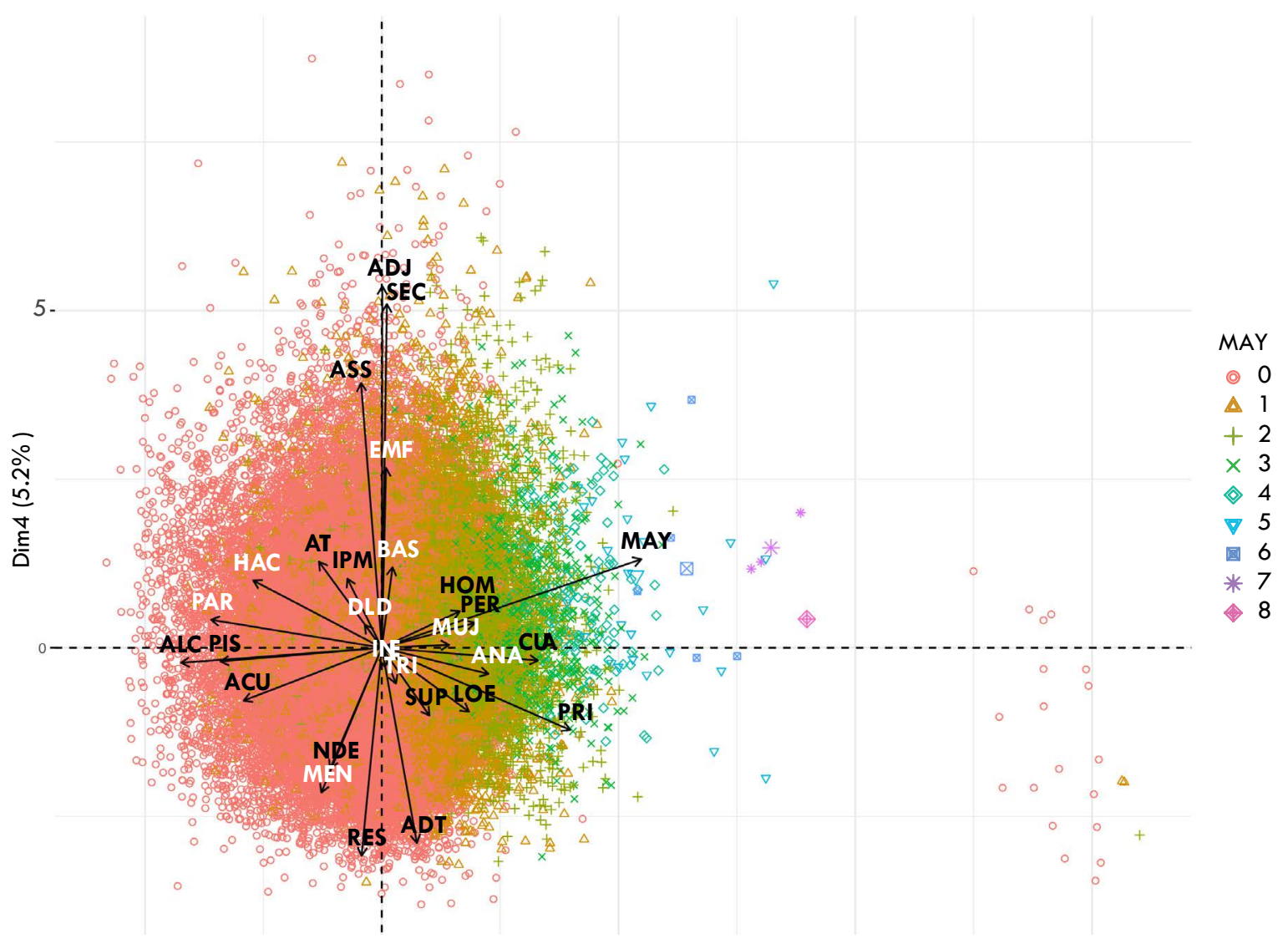

El segundo resultado relevante es que, en los hogares donde hay mayor cantidad de personas, hay tendencia a que sean más pobres. Esto lo corrobora el informe del Programa de las Naciones Unidas para el Desarrollo (PNUD, 2016), quien analiza cómo los hogares pobres tienen en promedio un mayor número de integrantes. García y Jaramillo (2019), al respecto, también infieren en sentido positivo, cuando indican que, los colombianos pasaron de 3,9 personas por hogar, en 2005, a 3,1 en 2018, con el fin de tener un control de la natalidad en los hogares vulnerables y bajar los índices de pobreza en el país. Se puede reflexionar sobre este comportamiento, cómo en hogares donde viven cinco personas y solo una trabaja es muy difícil mantener y sostener las necesidades básicas; este ejemplo es una situación muy común entre hogares pobres del país.
El tercer hallazgo por discutir se refiere a la condición de empleo formal, dado que en Colombia no tener un empleo formal aumenta la pobreza en los hogares. Al respecto, afirmaba Moreno (2009) hace más de una década:

La pobreza que es fundamentalmente de ingresos, se encuentra altamente relacionada con las condiciones en las cuales las personas se vinculan al mercado del trabajo (...) si bien la situación de pobreza no está limitada al sector informal, el hecho de que el jefe del hogar se encuentre en el sector informal del mercado laboral tiene poco más del doble de riesgo, de que su hogar se encuentre en la pobreza con respecto a quienes se encuentran en el sector formal (p. 19).

Otro resultado importante por mencionar, el cual no se asocia a factores específicos de privación 
que condicionan la pobreza en el país, es que la región más pobre de Colombia es la Caribe, cuyo planteamiento es ratificado en el estudio de Marrugo et al. (2015), quienes realizan un estudio de los determinantes de la pobreza en la región Caribe, y afirman que el país ha mostrado estar caracterizado por un modelo centro-periferia en perjuicio de las regiones Caribe, Pacífica, como las más pobres del país. Estos autores sustentan que las variables del mercado laboral y la tasa de desempleo están entre los principales determinantes de la pobreza en las regiones costeras de Colombia.

Por todo lo anterior, los resultados de este estudio ratifican que analizar la pobreza implica evaluar diversas dimensiones, porque desde cada una de ellas se pueden diseñar estrategias de política pública dirigidas que aporten a la reducción de la precariedad en las condiciones de vida de las personas y sus hogares. En este sentido, los resultados de este estudio son acordes con la inferencia de Dong et al. (2021), quienes afirman, "la reducción de la pobreza es un proceso sistemático y complejo, que involucra disciplinas como la economía, política, cultura, geografía, sociedad y psicología, y cambia con diferencias en el espacio, el tiempo y el entorno de desarrollo" (p. 146).

\section{Conclusiones}

Desde un punto de vista interpretativo, las conclusiones de este estudio se resumen en tres aspectos:

a) Para caracterizar la pobreza en Colombia, cuando se analizan las dimensiones del IPM de los hogares junto a variables sociodemográficas de las personas que los componen, se observa que estas últimas resultan más determinantes de la condición de ser pobre o no. Los factores de las viviendas, como tener un inadecuado material de paredes o los pisos, resultan poco relevantes.

b) Los datos del IPM (DANE, 2019) muestran que, las características que generan mayores diferencias entre los hogares pobres y no pobres, son el número de integrantes del hogar, su logro educativo y el acceso al mercado del trabajo mediante un empleo formal. c) Por regiones, se observan diferencias estadísticamente significativas entre las condiciones de pobreza multidimensional de los hogares, registrándose las mayores precariedades en las zonas costeras del país (regiones Caribe y Pacífica) y la Amazónica (incluyendo la Orinoquía).

Desde un punto de vista metodológico, es preciso concluir:

a) A pesar de que en este estudio no se utilizaron herramientas de georreferenciación para examinar la distribución espacial del IPM en Colombia, los datos DANE de este indicador permitirían plantear investigaciones académicas de este tipo, desde las cuales se pueda predecir la incidencia de la pobreza a nivel local.

b) Las técnicas de análisis multivariante (como el PCA utilizado en esta investigación), son útiles para exploraciones descriptivas en las que no se busque probar hipótesis de dependencia entre variables, sino que se estudie la interdependencia existente entre múltiples factores o condicionantes de un fenómeno socioeconómico, como es el de la pobreza.

c) Para investigaciones futuras acerca del estudio de la pobreza desde análisis cuantitativos, se recomienda evaluar variables que describan a las personas y sus posibilidades de tener acceso a la educación y el empleo formal; ya que estas condiciones son las que más pueden contribuir a la generación de estrategias de política pública que hagan frente a esta problemática social.

Dado que entender las características que delimitan la pobreza de una población permite diseñar acciones de política pública que contribuyan al mejoramiento de la calidad de vida de las personas, para terminar, es preciso indicar que, las dos principales recomendaciones de este estudio en términos de acciones gubernamentales requeridas, son:

a) Es necesario que el gobierno nacional apoye las estrategias que motivan a los colombianos a estudiar y prepararse académicamente, porque con ello indirectamente se evita la perpetuación de patrones de pobreza. 
b) En este estudio, se pudo corroborar que el acceso al mercado del trabajo es uno de los factores más determinantes de la condición de ser pobre o no; por tanto, se requieren mayores acciones e inversiones de los go- biernos locales (Gobernaciones y Alcaldías), para favorecer la empleabilidad formal en los territorios. Al igual que estrategias de apoyo a las microempresas, ya que estas ayudan a generar puestos de trabajo formal.

\section{Referencias}

Alkire, S. y Foster, J. (2007). Counting and multidimensional poverty measurement (OPHI Working Paper 7). University of Oxford. https://ophi.org.uk/working-paper-number-07/

Arias-Ramírez, R., Sánchez-Hernández, L. y Rodríguez-Morales, M. (2020). Pobreza y desigualdad en Costa Rica: Una mirada más allá de la Distribución de los Ingresos. Revista Estudios del Desarrollo Social: Cuba y América Latina, 8(1), 1-26. http://www.revflacso.uh.cu/index.php/EDS/article/view/422

Barahona, I. (2018). Poverty in Mexico: Its relationship to social and cultural indicators. Social Indicators Research, (135), 599-627. https://doi.org/10.1007/s11205-016-1510-3

Cardona, D., González, J., Rivera, M. y Cárdenas, E. (2014). Aplicación de la regresión lineal en un problema de pobreza. Interacción, 12, 73-84. https://doi.org/10.18041/1657-7531/interaccion.0.2315

Chacón-Mejía, C., Mattei, L. y Ramírez-Chaparro, M. (2021). Ruralidades en américa latina una mirada multidimensional de la pobreza a partir del análisis de componentes principales. Revista Visión Contable, (23), 133-155. https://doi.org/10.24142/rvc.n23a6

Congreso de la República de Colombia. (2011, 3 de noviembre). Decreto 4160. Por el cual se crea la Agencia Nacional para la Superación de la Pobreza Extrema, se determinan sus objetivos y estructura orgánica. http://www.suin-juriscol.gov.co/viewDocument.asp?id=1542859

Congreso de la República de Colombia. (2016, 21 de junio). Ley 1785. Por medio de la cual se establece la red para la superación de la pobreza extrema - red unidos y se dictan otras disposiciones. http://www. suin-juriscol.gov.co/viewDocument.asp?ruta=Leyes/30021681

Congreso de la República de Colombia. (2019, 8 de enero). Ley 1948. Por medio de la cual se adoptan criterios de política pública para la promoción de la movilidad social y se regula el funcionamiento del programa Familias en Acción. https://dapre.presidencia.gov.co/normativa/normativa/LEY\%201948\%20 DEL\%208\%20DE\%20ENERO\%20DE\%202019.pdf

Corbelle-Cacabelos, F. y Troitiño-Cobas, A. (2021). Pobreza multidimensional en España. Una aplicación de la metodología de Alkire y Foster. 2008 - 2015. Revista de Métodos Cuantitativos para la Economía y la Empresa, 31, 55-103. https://doi.org/10.46661/revmetodoscuanteconempresa.3489

Cuadras, M. (2014). Nuevos Métodos de Análisis Multivariante. CMC Edition. https://www.academia. edu/81 19269/NUEVOS_M\%C3\%89TODOS_DE_AN\%C3\%81LISIS_MULTIVARIANTE

DANE -Departamento Administrativo Nacional de Estadística-. (2018). Algoritmo para la construcción del indice de pobreza multidimensional - IPM. DANE. http://catalog.ihsn.org/index.php/catalog/7103/ download/83844

DANE -Departamento Administrativo Nacional de Estadística-. (2019). Boletín Técnico Pobreza multidimensional en Colombia. DANE. https://www.dane.gov.co/files/investigaciones/condiciones_vida/ pobreza/2018/bt_pobreza_multidimensional_18.pdf

DNP -Departamento Nacional de Planeación de Colombia-. (2012, 28 de mayo). Conpes Social 150. https:// colaboracion.dnp.gov.co/CDT/Conpes/Social/150.pdf

Dong, Y., Jin, G., Deng, X. y Wu, F. (2021). Multidimensional measurement of poverty and its spatio-temporal dynamics in China from the perspective of development geography. Journal of Geographical Sciences, 31 , 130-148. https://doi.org/10.1007/s11442-021-1836-x 
Dorian, L. (2017). Metodología Alkire y Foster en la medición de Pobreza Multidimensional: el caso colombiano (MPRA Paper No 80102). MPRA. https://mpra.ub.uni-muenchen.de/id/eprint/80102

Fagnola, B. y Moneta-Pizarro, A. (2021). Identificación de la pobreza multidimensional en Argentina con métodos robustos de análisis factorial. Cuadernos del CIMBAGE, 1(23), 1-20. http://157.92.136.232/index.php/ CIMBAGE/article/view/2053

Fuentes, C., Peña, S. y Hernández, V. (2018). La medición multidimensional de la pobreza a nivel intraurbano en Ciudad Juárez, Chihuahua (2012). Estudios fronterizos, 19, 1-25. https://doi. org/10.21670/ref.1801001

Galindo, M. P. (1986). Una alternativa de representación simultánea: HJ-Biplot. Qüestiió, 10(1), 13-23. http://hdl. handle.net/2099/4523

Gamboa, G., Mingorría, S. y Scheidel, A. (2020). The meaning of poverty matters: Trade-offs in poverty reduction programmes. Ecological Economics, 169, 106450. https://doi.org/10.1016/j.ecolecon.2019.106450

García, D. M. y Jaramillo, Á. (2019). El cambio social que se gesta en los hogares colombianos. https://razonpublica. com/el-cambio-social-que-se-gesta-en-los-hogares-colombianos/

González, J. (2019). Aumento de la pobreza en Colombia no debe subestimarse. https://razonpublica.com/aumentode-la-pobreza-en-colombia-no-debe-subestimarse/

Hotelling, H. (1933). Analysis of a complex of statistical variables into principal components. Journal of Educational Psychology, 24(6), 417-441. https://doi.org/10.1037/h0071325

Marrugo, C., Del-Risco, K., Herrera, J. y Pérez, G. J. (2015). Determinantes de la pobreza en la región Caribe colombiana. Revista de Economía del Caribe, (1 5), 47-69. http://dx.doi.org/10.14482/ecoca.15.7192

Mayorga-Henao, J. M., Hernández, L. M. y Lozano, M. C. (2021). Segregación y pobreza multidimensional en el sistema urbano colombiano. Bitácora Urbano Territorial, 31(2), 113-129. https://doi.org/10.15446/bitacora. v3 $\ln 2.89600$

Moreno, J. A. (2009). Informalidad Laboral y Pobreza Urbana en Colombia (Documento de trabajo 134). CIDSE, Universidad del Valle. http://biblioteca.clacso.edu.ar/Colombia/cidse-univalle/20121113055828/ DocTrab_134.pdf

Munévar, W. (2019). Pobreza, exclusión social y comportamiento económico de los países latinoamericanos. Revista Observatorio de la Economía Latinoamericana, (2019), 1-16. https://www.eumed.net/rev/oel/2019/02/ pobreza-exclusion-social.html

OCDE -Organización para la Cooperación y el Desarrollo Económico-. (2016). Educación en Colombia. OECD. https://www.mineducacion.gov.co/1759/articles-356787_recurso_1.pdf

Pearson, K. (1901). LIII. On lines and planes of closest fit to systems of points in space. The London, Edinburgh, and Dublin Philosophical Magazine and Journal of Science, 2(1 1), 559-572. https://doi.org/10.1080/14786440109462720

Peña, D. (2002). Análisis de datos multivariantes. https://www.researchgate.net/publication/40944325_Analisis_ de_Datos_Multivariantes

PNUD -Programa de las Naciones Unidas para el Desarrollo-. (2016). Contexto y caracterización de los hogares en situación de pobreza en Colombia. PNUD. https://info.undp.org/docs/pdc/Documents/COL/ Investigaci\%C3\%B3n_Caracterizacio\%CC\%81 n\%20de\%20los\%20pobres_LAR_Sin\%20publicar.pdf

Rajeev, M. (2012). Human rights indicators: a guide to measurement and implementation. United Nations Human Rights. https://www.ohchr.org/documents/publications/human_rights_indicators_en.pdf

Sacchidanand, M. y Biswas, S. (2017). The role of education in poverty alleviation: Evidence from Bangladesh. Journal of Economics and Sustainable Development, 8(20), 151-160. https://www.iiste.org/Journals/index.php/ JEDS/article/view/39320/40429

Tejero-Pérez, A. (2018). Pobreza laboral en España. Un análisis dinámico. Revista Internacional de Sociología, 76(2), e096. https://doi.org/10.3989/ris.2018.76.2.16.54 